\title{
World Food Day
}

16 October is World Food Day, and against a backdrop of increasing world hunger, we look at the role of science in making food production more distributed and more sustainable.

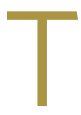
he widespread availability of food is arguably the most important cornerstone for the development of society. The successive agricultural revolutions transformed society from hunter-gatherers to agricultural communities, and subsequently allowed the increased population and urbanization levels that we see today. And we have become accustomed to gradual improvements in our quality of life, with slow but continual decreases in such areas as extreme poverty and hunger taking place year-on-year. Indeed, one of the UN Sustainable Development Goals is the challenging but laudable effort to reach zero hunger by 2030 (www. un.org/sustainabledevelopment/hunger). Startlingly, however, these trends have recently reversed and, according to the most recent Global Report on Food Crises ${ }^{1}$, hunger has been increasing for the past three years. As we approach World Food Day, it is worth looking at food production, especially the role of science in it.

First, it should be pointed out that many of the causes of hunger are political rather than technological in nature. The aforementioned food crises report cites "new or intensified and protracted conflict" as one of the main causes of the increase in global hunger ${ }^{1}$ - a dramatic example of the effect that the current worldwide political instability has on large swathes of the world population (over 11 million more people required urgent humanitarian action in 2017 compared with 2016). Indeed, the problem is not simply a global food shortage: despite the increase in hunger, the amount of food produced worldwide (measured in calories per capita) has increased. Given these facts, it seems clear that methods for increased production on a worldwide scale - no matter how welcome they will be - will not solve the issue of food inequality. Rather, we need methods for food production and protection that can be distributed more evenly across the globe, and critically these methods must be applicable in regions without the infrastructure that is taken for granted in most countries with food surpluses. Furthermore, we must ensure that any developments are cost effective thereby ensuring that they are available to the people who need them the most - and enforceable without increasing the demands we already place on the planet.

\section{We need methods for food production and protection that can be distributed more evenly across the globe, and critically these methods must be applicable in regions without the infrastructure that is taken \\ for granted in most countries with food surpluses.}

Taking the twin points of sustainability and cost, we have an area where catalysis plays a vital role. No discussion of modern agriculture would be complete without mentioning the Haber-Bosch process. While many factors were responsible for the sudden rise in the human population from the start of the twentieth century, large-scale ammonia production was a necessary - if not sufficient - step. It has been poetically described as a method that "turns air into bread" ${ }^{2}$, though admittedly this does skip over some of the challenges that researchers in this field face. However, these benefits come with a cost - huge amounts of energy are required to turn stable $\mathrm{N}_{2}$ into the desired $\mathrm{NH}_{3}$ (nitrogen fertilizer is responsible for over half of the fossil fuel use in agricultural primary production ${ }^{3}$ ). This results in not only increased emissions of greenhouse gases but also increased costs, meaning that fertilizer can be expensive and therefore underused by smallholder farmers in developing countries, leading to reduced crop yields.

Many research avenues are being explored in parallel in order to make nitrogen reduction more energy efficient ${ }^{4}$. While this is vital, reducing the energy input for the ammonia synthesis on its own is not enough. The hydrogen feedstock that is used to reduce molecular nitrogen typically comes from steamreforming of natural gas, meaning that in effect we are turning fossil fuels rather than air - into bread. This is clearly not sustainable. Photo- and electrocatalytic water-splitting offer a way to decouple hydrogen generation from fossil fuels, though more work is needed to increase efficiencies and stabilities of the catalysts for these processes while also lowering costs.

This leads into another key area in of production: delocalization (you can find a more extensive discussion on delocalization in sustainable development in the Comment by Resasco, Wang and Sabatini in this issue). The increasing viability of photo- and electrochemical means of chemical production open the possibility to move away from the large plants traditionally used for the synthesis of ammonia and other commodity chemicals. Bearing in mind that many smallholder farmers (who make up the overwhelming majority of farmers in developing countries ${ }^{5}$ ) operate in locations with poor transport infrastructure, the decentralization of production could simultaneously increase availability and reduce costs on a local level.

Other important areas for chemistry are the development of veterinary medicines and plant protection products (such as pesticides and herbicides). These come with an environmental cost, and overuse can have severe negative effects on soil, water and wildlife. However, they also play a vital role in helping to feed the planet. It is estimated that $42 \%$ of worldwide achievable production is lost due to pests and disease, which would increase to $70 \%$ if current methods of crop protection were removed ${ }^{6}$ (these numbers may seem unreasonably high, but it should be noted that 'achievable production' is defined as the maximum output possible, not the current nor the economically optimal level of production). In order to maintain crop yields while reducing environmental impact, more-selective products are needed, in combination with a much greater precision in their application. And perhaps new areas of research in chemistry and biology will offer strategies for crop protection that avoid the need for the spraying of synthetic biocides altogether.

With population increase of over two billion people expected in the next 30 years, great changes are needed if we are to have any hope of reducing hunger to zero. Political solutions to man-made catastrophes are necessary, as are technological advancements to allow us not only to feed 10 
billion people, but to do so in a manner that does not end up destroying our planet.

Published online: 11 October 2018

https://doi.org/10.1038/s41929-018-0175-5
References

1. Global Report on Food Crises (Food Security Information Network, 2018).

2. Hager, T. The Alchemy of Air (Random House, New York, 2009).

3. The State of Food and Agriculture (Food and Agriculture Organization of the United Nations, 2017).
4. Foster, S. L. et al. Nat. Catal. 1, 490-500 (2018).

5. Altieri, M. A. \& Koohafkan, P. Enduring Farms: Climate Change, Smallholders and Traditional Farming Communities (Third World Network, 2008).

6. Bourguignon, D. EU Policy and Legislation on Pesticides (European Parliamentary Research Service, 2017). 